\title{
The Design and Implementation of Computer Hardware Assembling Virtual Laboratory in the VR Environment
}

\author{
Shaoming Sun ${ }^{1}$, Tianwei $\mathrm{Xu}^{2, \mathrm{a}}$ and Juxiang $\mathrm{Zhou}^{1}$ \\ ${ }^{1}$ Key Laboratory of Educational Information for Nationalities, Ministry of Education, Yunnan Normal University, Kunming, 650500, Yunnan, \\ China \\ ${ }^{2}$ Graduate Student Department, Yunnan Normal University, Kunming 650500, China
}

\begin{abstract}
In solving the problems of slowly updated laboratory equipment, heavy hardware wastage and potential danger in the course of traditional computer hardware assembling experiment, this article proposes a design and concrete implementation of virtual laboratory for computer hardware assembling based on immersive VR environment. Adopt 3Ds Max and Unity3d software to create 3D models and build the scene, and Mojing SDK as the VR display effect and the tool of interactive interface, the article achieved the design and implementation of virtual laboratory application in mobile devices. Current virtual laboratories based on Virtools can only run in Windows environment, and this design overcomes such limitation. In addition, with rich scenes and tutorial, this design combines the headmounted display and somatosensory controller to greatly promote the immersion and interactivity, thus enhancing the interests of students. This is a new trail to improve the traditional experimental teaching effect.
\end{abstract}

\section{Introduction}

Virtual reality technology, also known as soul technology, is based on computer technology and uses relevant science and technology to form a virtual environment similar to the surroundings. In this virtual environment, learners can do whatever they want to do in the real world[1]. Virtual reality has three characters: interaction, immersion and imagination. According to the degree of immersion and interaction, virtual reality can be generally divided into semi-immersive desktop virtual reality system and immersive virtual reality system[2]. The semi-immersive desktop virtual reality system mainly relies on tools like 3D modelling provided by computer to create a screenbased virtual environment. Users can realize humancomputer interaction through input tools such as mouse and keyboard. The advantages of this system lie in its low cost and high penetration rate[3]. The disadvantage is the poor immersion effect caused by the influence of the operating environment. The immersive virtual reality system refers to completely closing the visual, auditory and tactile sense by using a head-mounted display, data gloves or other handheld devices and thereby achieving a good immersive experience.

With the development of virtual reality technology, especially in the first year of VR, well-known companies such as Google, Facebook, HTC, Xiaomi, and Storm have directly or indirectly launched their VR products and plans. Virtual reality has evolved from computer simulation to immersive virtual reality, undoubtedly providing more technical support for the application of virtual reality in education. Its application in education is realized by students learning in a three-dimensional virtual environment presented by virtual reality related hardware and software, whose application value is mainly reflected in four aspects: firstly, creating a more realistic learning scenario for learners and increases their learning experience through multisensory interaction[4]; secondly, enhancing learners' motivation and participation[5]; thirdly, allowing learners to learn independently; lastly, bridging the gap between theory and practice. In recent years, virtual reality technology has been widely used in various teaching fields such as physics, chemistry, biology, history, medicine, agriculture, dance and aerospace, playing a significant role in assisting and promoting students' learning as well as providing a new way to improve the traditional teaching models.

At present, the application of virtual reality technology in education has achieved remarkable results. However, compared with foreign technology and application level, domestic practice research is still on its initial stage[6]. In Chinese education domain, most theoretical and practical studies are based on desktop virtual reality system (Desktop-VR) with its interactivity and immersion to be improved. Taking computer hardware assembly course as an example, many scholars have conducted related researches, such as Yan Lina of Sichuan Normal University, Ge Qiaoyan of Zhejiang University of Technology and Li Qiang of Bohai University, proposing computer hardware assembly virtual labs based on 3Ds Max and Virtools technology. On the whole, however, due to the limitations of development technology and

a Corresponding author: xutianwei@ynnu.edu.cn 
hardware, the virtual labs only support running in the Windows environment and require learners to interact with their objects through keyboard and mouse during the learning process, lacking immersion and interactivity and leading to insufficient interest in learning, even though they meet learners' basic learning needs. This study adopts Unity3d technology as the development platform, Mojing SDK as the development tool for VR effects, smart phone as the operating environment for virtual labs and storm mirror and daydream sensor handle as virtual reality display and interactive devices, building a computer hardware assembly virtual lab in a immersive VR environment to solve the previous problems that computer hardware assembly virtual laboratory is not highly immersive and cannot create real teaching situations which lead to low learning efficiency.

\section{Design of virtual laboratory in the VR environment}

\subsection{Teaching content design}

Computer Hardware Assembly is one of the compulsory courses for students majoring in computer application in secondary vocational and technical schools, playing a vital role in the whole process of discipline development. Through the study of this course, learners can have a better understanding of the basic knowledge of computer hardware and improve their practical ability to assemble computers. Therefore, the computer hardware assembly virtual laboratory is based on the teaching objectives of the computer hardware assembly of the secondary vocational school. Different presentation methods are utilized according to the difficulty level of knowledge points[7]. When designing the teaching content of hardware's names, functions and parameters, the three-dimensional model and text are used to increase the perceptual intuition of teaching given that the teaching content of this part is mainly to enable learners to correctly understand the names, functions and parameters of the computer hardware. In terms of simulative assembly, the video demonstration is used at first to help students make clear of the points for attention in the process of computer hardware assembly and master how to properly assemble a computer, considering that this is the core part of the entire virtual laboratory. After watching the demonstration video, learners are asked to start the installation situation using somatosensory handle with an aim to improve their practical ability. When it comes to designing the teaching content of common hardware faults, students are supposed to be familiar with them and corresponding solutions to enhance their problem-solving ability. Therefore, the teaching content is presented in the form of text.

\subsection{Functional module design}

Based on the above analysis of the teaching contents, the functional module of the virtual laboratory is divided into three ones: basic learning module, virtual experiment module and experimental feedback module. The specific function introduction is shown as follows.

\subsubsection{Basic learning module}

The basic learning module can be divided into two submodules: hardware model display and hardware function introduction. The main function of the hardware model display module is to enable learners to roam in the virtual world from the first-person perspective through the somatosensory handle interaction device and observe the basic structure of each hardware from any angle, greatly enhancing learning's intuitiveness and making up for the teaching problem that the students can't observe the computer hardware up close and for a long time due to the lack of hardware equipment in the traditional experimental teaching. The hardware function introduction module introduces the basic functions of hardware and the current mainstream parameters. Students can not only know the basic functions and parameters of hardware, but also able to apply the knowledge in daily lives after learning, so that they can understand the computer performance based on the configuration list when they purchase a computer in the future[8]. When certain hardware is updated over time, administrator can adjust the teaching content in the system as needed to ensure its cutting edge.

\subsubsection{Virtual experiment module}

This module is the core part of the entire virtual lab, consisting of two sub-modules: assembly video demo and simulative assembly. The teaching content of the assembly video demonstration is to produce a teaching video of computer hardware assembly based on learning theory and teaching theory after analysing the textbook Computer Hardware and Assembly Maintenance and learner characteristics majoring in computer application of secondary vocational schools, helping them understand the correct installation steps and precautions so as to improve the scientificity and standardization of the simulative assembly process. Learners can also watch the video after simulative assembly as needed and find out the wrong operations during the assembly process. This part revoles around learners' simulating to assemble a computer and creating a virtual learning environment through virtual reality technology to get a sense of presence. Learners operate the somatosensory handle during the learning process and receive corresponding feedbacks. If correct, the selected computer hardware will move to the corresponding position of the motherboard and the system will give feedback on the correct installation; if wrong, the system will present corresponding prompt message to help students complete the experimental task smoothly. The experimental results are significantly better than some existing computer hardware assembly virtual labs.

\subsubsection{Experimental feedback module}

This module is mainly composed of two sub-modules: common fault cases and experimental tests. Common fault case module is designed to introduce high-frequency faults 
of the computer hardware, such as loss internal storage, and the corresponding solutions, assisting students to development a solution in their minds and deal with it alone when they encounter a hardware failure as well as improve their practical ability to solve problems. In terms of the experimental test module, a test is required after the studying the basic learning module and the virtual experiment module. The content of the exercises is based on the teaching objectives and content, going from the easy to the difficult and complicated, largely increasing students' self-confidence and enhance their learning effect. They can evaluate their own learning results after the test and fill in the gaps of knowledge.

\section{Implementation of Virtual Laboratory Based in the VR Environment}

\subsection{The construction of development environment}

This virtual lab uses Unity3d as the main development environment in software, Mojing SDK as a development tool for virtual reality and 3Ds MAX as a development tool for 3D models.

First, create a new project file in Unity3d software and a new Assets folder in the project directory used to store Mojing SDK, 3D models, image, audios and videos. In this way, we can dynamically watch scenes' finish effect by calling the memory loading mechanism configured by Unity $3 \mathrm{~d}$ at any time during the development process with an aim to improve the loading speed and running performance of scenes.

Then, import the Mojing SDK into the project file through Import Package under the Assets menu bar, delete the original Main Camera in the scene, create a new MojingMain as the main camera in the virtual lab, and then utilize the Import New Assets under the Assets menu to import all the resources needed into the project.

Finally, for each function page, we create multiple scenes, skyboxes, and ground systems to load its teaching content. This is the core content of the entire virtual lab. Command SceneManager. Load Scene to realize the switches between scenes.

\subsection{The realization of the main functional modules in the virtual laboratory}

According to the design of above functional modules, this essay divides the virtual lab into various learning modules so that learners can learn the teaching content in the corresponding modules according to their actual needs. In the development process of the virtual lab, each experimental function module is packaged in a separate scenario and linked by the hierarchical script so that learners can selectively study as required. The specific implementation of each scenario is as follows:

\subsubsection{Main menu module}

As soon as learners enter the virtual lab, they first come across the menu interface (as shown in Figure 1). Considering that the use objects of virtual lab are students majoring in computer application from secondary vocational school, the design of the interface layout adheres to the principle of integrity and navigation. Integrity refers to whether the UI button is consistent with the background colour in the scene and whether learners feel the entire scene interface is a whole during use, so as to avoid unnecessary interference distracting learners' attention. Navigation refers to whether the set UI button can navigate accurately and ensure learners clearly choice the corresponding learning module according to their actual needs without confusion. A total of four UI interactive buttons are set in the main menu interface, correspondingly Basic Learning, Experimental Assembly, Experimental Feedback, and Experimental Help. Experimental Help provides a guide for learners who use the virtual lab for the first time and make them clear of the precautions during use to help learner can better them better finish the learning tasks. Learners can choose the corresponding learning module through gaze interaction. In terms of realizing gaze interaction, first we should import the UI resources made in Photoshop CS6 into the Unity project file, create a canvas in the scene where UI is placed, add a Box Collider to UI for collision detection and script code that triggers events to the UI and HeadCtrl in the Canvas for gaze interaction. The core script code is as follows:

private void SetSelectedNode()

\{

var forward = mainCamera.transform. forward;

if (Physics.Raycast(transform.position, forward, out hit, 000))

currentNode $=$ hit.collider.gameObject.GetComponent $<$ Basiccontrol $>()$;

\} \}

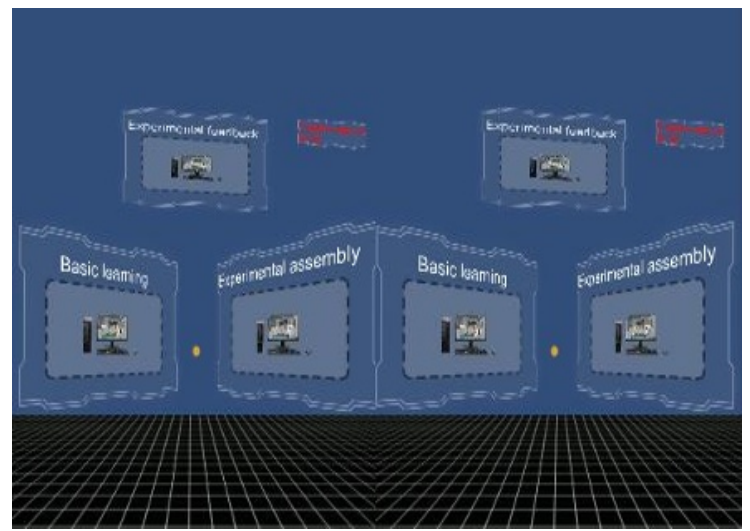

Figure 1. Main menu interface

\subsubsection{Basic learning module}

The main function of basic learning module is to create a highly immersive and interactive virtual learning environment for learners. During the learning process, 
learners can use the somatosensory handle to wander in the scene from a first-person perspective and observe the hardware model from any angle according to their own needs (as shown in Figure 2), which can greatly make up for the teaching problem in the traditional experimental teaching process that students cannot observe a certain hardware at a close distance and for a long time due to the lack of hardware equipment. Learners can interact with each hardware model through the head-controlled gaze interaction during the learning process and form a preliminary concept in their minds about name, function, basic parameters and other related knowledge of the hardware (as shown in Figure 3) to carry out computer simulation assembly according to different hardware functions. If students want to learn certain hardware again, they can push the trigger button on the somatosensory handle to go back and study it repeatedly.

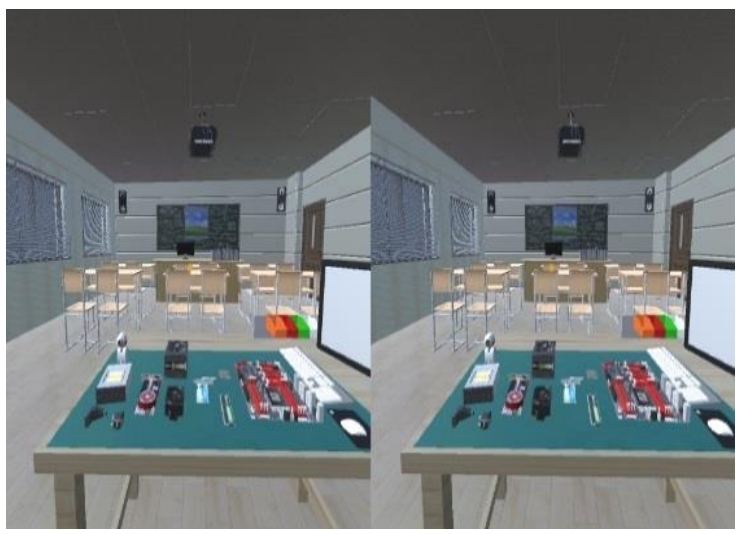

Figure 2. Basic learning scene interface

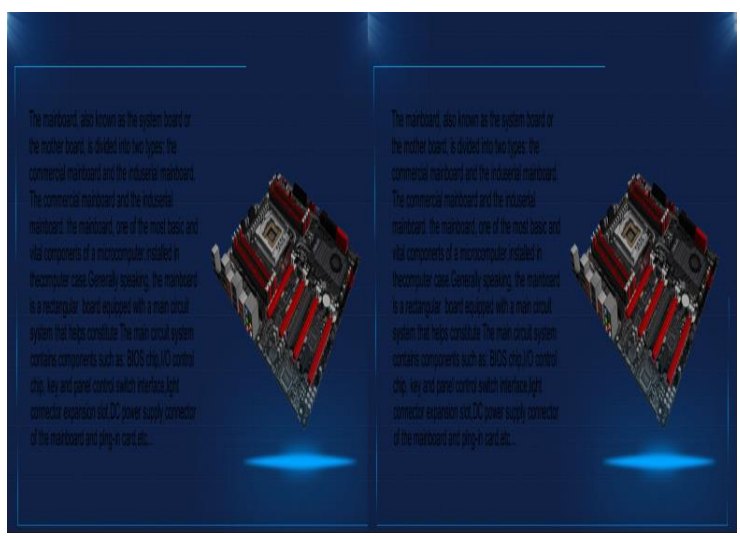

Figure 3. Hardware introduction interface

Virtual tour is about making use of the displacement of the virtual camera to realize the effect of scene exploration. To be honest, to achieve virtual exploration is to form a mapping relation between the data of the smartphone accelerometer sensor and four directions of the virtual camera displacement, namely, front, back, left and right. First, create a Character Controller in the scene and add script code to it. Apply Controller.Move in the Update function to dynamically update the controller's location in the script and Input.acceleration method to obtain data of the phone transmitter. This method is mainly used to get the sub-scales of $\mathrm{X}$ axis, $\mathrm{Y}$ axis and $\mathrm{Z}$ axis of the mobile phone, which are represented by Input.acceleration.x, Input.acceleration.y and Input.acceleration.z. During the roaming process, the character controller cannot advance if it collides with other objects in the scene and only turn left, right or back to avoid penetrating other objects and to enhance the immersive experience. This function is realized by object engine provided by Unity $3 \mathrm{~d}$ to add a Collider to the virtual camera and 3D model in the scene. The core script code of scene roaming function is as follows:

private void UpdateFunction()

\{

Vector3 moveDistance $=$ Vector3.zero; if (MoveWithPlatform())

Vector3newGlobalPoint=movingPlatform.activePlatfor m.TransformPoint(movingPlatform.activeLocalPoint); moveDistance $=($ newGlobalPointmovingPlatform.activ e-GlobalPoint); if (moveDistance != Vector3.zero) controller.Move(moveDistance); \} \}

\subsubsection{Virtual experiment module}

The virtual experiment module consists of two parts: assembly video demonstration and simulative assembly. As for assembly video demonstration, some precautions and specific operating steps in the process of assembling computer hardware in the real environment are presented in the form of video. During use, learners can first interact with the 3D model of the blackboard in the lab through the ray emitted by the somatosensory handle. When the collision event is triggered, the teaching video of computer assembly will be played, forming a preliminary concept on learners' minds about how to install the machine correctly before the simulative assembly and laying a theoretical foundation for the subsequent simulative assembly. After watching the video, learners can simulate the installation by themselves. Given that the simulation assembly is a core part of the entire virtual laboratory, the design of the whole scene follows the principle of education, science and art. The quality of education and science are mainly reflected in the design of the content based on the teaching objectives in line with students' cognitive rules. The artistic nature is reflected in the layout, colour and light of the scene interface to arouse students' interest. Learning interest. In this part, a highly immersive virtual experiment environment is created to guide students to complete the simulative assembly experiment based on the theoretical knowledge previously learned. Taking the simulative installation of CPU as an example, all the hardware models on the console can be detected by using a somatosensory handle to control the direction of rays (as shown in Figure 4). Detecting the 3D model of Hardward means determining the desired hardware. By this time, the system records the current location of the detected hardware and the name of the data and takes the hardware as the launch point to re-emit the ray to the correct position of the motherboard. The ray will detect the collider set on the main board and feed back the corresponding Tag data to 
the API in the script. When the value obtained on the hardware is judged by the value obtained at a certain position on the main board and matches the condition, the hardware will move with the ray to the correct position on the motherboard and give correct feedback. If wrong, the corresponding prompt is given to help users successfully complete the experimental task (as shown in Figure 5). The core script code is as follows:

void FindTarget() \{
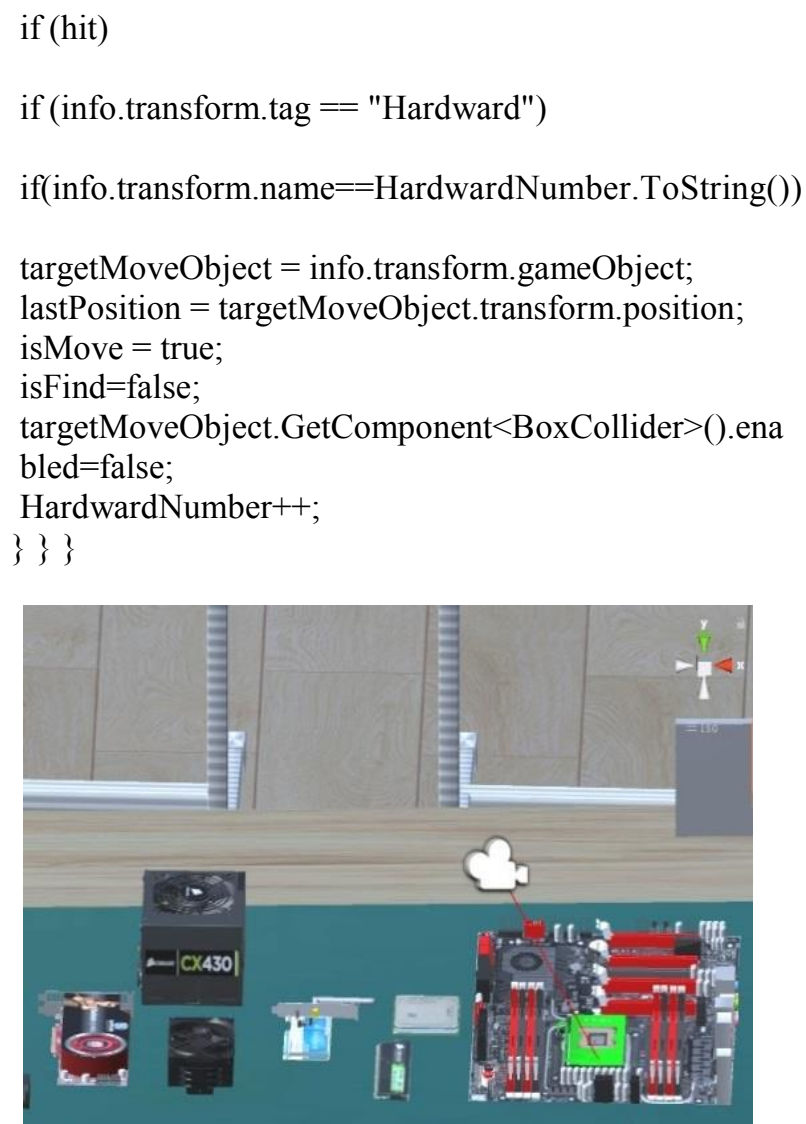

Figure 4. Gamma ray detection interface

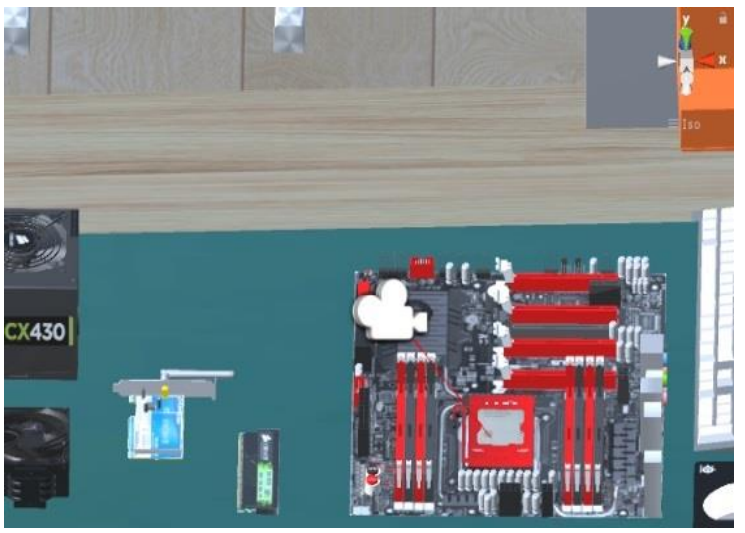

Figure 5. Simulated assembly interface

\subsubsection{Experimental feedback module}

Experimental feedback module is a supplement and check after studying the first two modules, mainly consisting of common fault cases and experimental tests. The common hardware failure module is mainly presented in the form of classification, so that learners can learn more common faults of hardware in the learning process without confusion. This module is realized by controlling the ray direction by a somatosensory handle to collide with each $\mathrm{UI}$ in the scene and enter the corresponding learning unit. The effect is shown in Figure 6. The design of the experimental test module abides by the principle of openness, not only telling right or wrong but also providing more space for reflection and improvement, because effective feedback should be oriented and illuminating and indirectly solve some specific problems encountered by students during the experiment. Given that the presenting form is exercise and learners may take some notes in the learning process, this part is mainly presented in the form of single screen and interacts in the form of touch screen. The effect is shown in Figure 7.

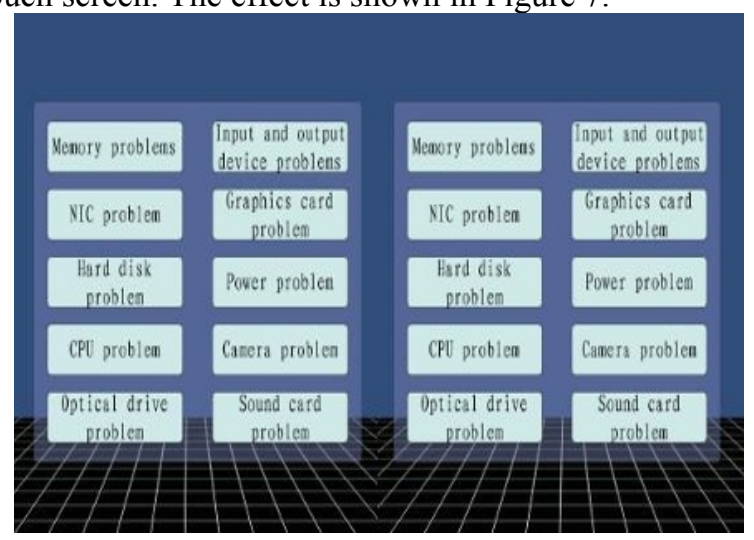

Figure 6. Common fault case interface

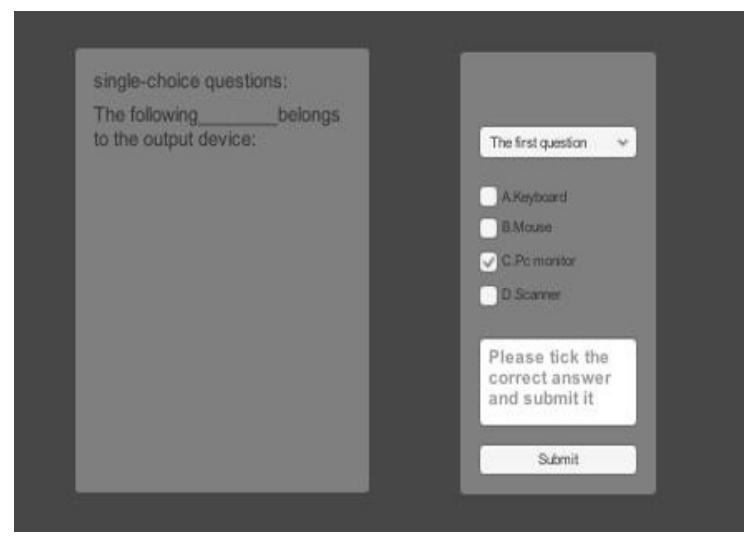

Figure 7. Experimental test interface

\section{Conclusion}

In this paper, a virtual laboratory of computer hardware assembly is built in the VR environment based on the training needs of computer hardware assembly experiment teaching in traditional environment and by combining 3Ds Max and Unity3d technologies. The virtual laboratory uses VR glasses and somatosensory handles as the presentation platform and interaction mode so that learners can immerse themselves in the process. At the same time, the scene creation and teaching design of the three functional modules---basic learning module, virtual experiment module and experimental feedback enhance students' 
interest in learning, which not only can effectively make up for the shortcomings in the traditional experimental teaching process, but also solve the limitation that the existing virtual lab based on virtools technology can only run in Windows environment. In the subsequent researches, the interaction mode in virtual experimental process and the degree of refinement of the interaction operation will be further improved. In addition, AI speech recognition system will be taken into consideration so that learners can complete virtual experiments through multiple interaction modes and the authenticity and operability of the experiment will be improved. In this way learners can better complete the experimental tasks and improve their learning motivation and experience.

\section{References}

1. QinPing. Zhao, Scientia Sinica(Informationis) 39, 246 (2009)

2. Gengzhong. Zheng, Computer Systems \& Applications 2, 62-65 (2008)

3. Pei. Pei, The Chinese Journal of ICT in Education 8, 9-4-96 (2018)

4. Nan. Ding, Modern Educational Technology 27, 1925 (2017)

5. Yuan. Gao, Dejian. Liu, Zhenzhen. Huang, Huairong. Huang, e-Education Researchn 37, 77-87+103 (2016)

6. L. Benetazzo, M. Bertocco, F. Ferraris, et al., IEEE Transactions on Instrumenta-tion \& Measurement, 49, 349-356 (2000)

7. B. Spanlang, J.M. Normand, D. Borland, et al., Frontiers in Robotics \& Ai, 1,9 (2018)

8. E. Roy, M.M. Bakr, R. George,Saudi Dental Journal, 29, $41-47$ (2017) 